\title{
Thyroid Lobectomy
}

National Cancer Institute

\section{Source}

National Cancer Institute. Thyroid Lobectomy. NCI Thesaurus. Code C51649.

Surgery involving complete excision of a lobe of the thyroid gland. 